(C) 2016 IEEE. Personal use of this material is permitted. Permission from IEEE must be obtained for all other uses, in any current or future media, including reprinting/republishing this material for advertising or promotional purposes, creating new collective works, for resale or redistribution to servers or lists, or reuse of any copyrighted component of this work in other works. 


\title{
Quadrature Broadband Phase Shift Generation Using Passive RC Polyphase Filter for RF Front-end
}

\author{
Meriam Gay Bautista, Eryk Dutkiewicz, Xiaojing Huang, Diep Nguyen, Forest Zhu \\ University of Technology Sydney, Global Big Data Technology Centre, \\ Ultimo NSW 2007, Australia \\ Email: meriam.g.bautista@student.uts.edu.au; \{eryk.dutkiewicz, xiaojing.huang, diep.nguyen, forest.zhu\}@uts.edu.au
}

\begin{abstract}
This paper reviews the state of art of a polyphase complex filter for $R F$ front-end low-IF transceivers applications. We then propose a multi-stage polyphase filter design to generate a quadrature $I / Q$ signal to achieve a wideband precision quadrature phase shift with a constant $90^{\circ}$ phase difference for self-interference cancellation circuit for full duplex radio. The number of the stages determines the bandwidth requirement of the channel. An increase of $87 \%$ in bandwidth is attained when our design is implemented in multi-stage from 2 to an extended 6 stages. $A$-stage polyphase filter achieves $2.3 \mathrm{GHz}$ bandwidth.
\end{abstract}

Keywords: Phase shift, Polyphase filter, Quadrature, RF Front-end, $90^{\circ}$ phase

\section{INTRODUCTION}

Wireless communications traffic demand is expected to increase by more than 1000 -fold in the next decade. To serve this ever-increasing demand, it is of great interest for $\mathrm{RF}$ designers to design integrated circuits that are more power/energy efficient with smaller footprint and lower implementation cost [1]-[3]. Since it contains both analogue and digital circuitry, the integration of RF, analogue and baseband in a single chip has always been a critical challenge [4]. Emerging mobile communications (e.g., LTE-Advanced, 5G) demands for extremely high data rate (peaks at 10Gbps) and ultra-low latency (e.g., in order of ms or less) [5][6]. For many years, there have been several efforts in developing single wireless transceivers that integrates both transmitter and receiver on chip. Some of the pioneers who made their contributions in theory and practical implementation of transceivers on chip are Abidi [7], Lee [8] and Steyaert [9]. This led important improvements in design implementation and development of new circuit architecture [5].

The high volume of data traffic in the network will be a big challenge. Full Duplex (FD) technology is considered to be a key technique to potentially double the speed of wireless communication on between a transmitter and a receiver, allowing the transmission and reception of data on the same frequency band [10]. In achieving full duplex transmission, a radio has to completely cancel out the selfinterference that is present in the system. If self-interference is not completely cancelled, a residual interference reduces the signal to noise ratio (SNR), reducing the overall throughput of the Full Duplex radio and thus degrading the efficiency of the system [11].

Radio transceivers require a complex filter to eliminate unwanted signals. These are important building blocks in many analog front-end receivers [12]-[17]. Resistor-
Capacitor (RC) Polyphase filter (PPF) is one of the complex filters that can be implemented. Its RC network is suitable for high frequency design [22]. In radio frequency (RF) applications, the performance of passive polyphase filter in terms of image rejection and quadrature signal generation depends on the component mismatch [18][19].

There are various methods to generate the quadrature I/Q signal. For Low-IF architecture, resistor Capacitor polyphase filters are often more favourable thanks to their simple implementation and convenience for high frequency application where high level of integration is required [18]. The capability of RC polyphase filters to provide a required performance in a wide frequency range is critical for the application of double-quadrature low-IF architectures in a multi-standard receiver [24] and transceivers. Furthermore, polyphase filter can be used as a phase shifter by varying the output amplitude, while using a variable gain amplifier (VGA) and combining them to change the phase of the output signal.

This article presents the design consideration and performance of a multi-stage polyphase filter to generate a wide bandwidth quadrature I/Q phase shifter for selfinterference cancellation circuit for full duplex radio. The precise quadrature phase shifting is suitable for broadband low-IF RF front-end transceivers with interference cancellation mechanism. This design is simulated using BiCMOS $0.13 \mu \mathrm{m}$ process [28]. The rest of the paper is organized as follows. The concept of complex mixing and quadrature phase shift are in Section II. A polyphase filter design is adopted to generate the precise quadrature in Section III. The simulation results and comparison in Section IV and conclusions are drawn in Section V.

\section{COMPLEX MIXING}

One important issue in receiver design is to suppress any unwanted signal prior to mixing operation. This unwanted input signal band which will be superimposed on the desired signal after mixing is referred as the image signal band. An image rejection filter implements this process. To avoid image problem that is due to the positive and negative frequencies is to mix the signal with the complex exponential that can be express as

$$
e^{-j 2 \pi f_{L O} t}=\cos \left(2 \pi f_{L O} t\right)-j \sin \left(2 \pi f_{L O} t\right)
$$

The output of mixing the real signal to the complex exponential gives a shifted version of the real signal spectrum, due to this shifting mechanism it help to eliminate the image problem. Mixing of the two signals as shown in Fig. 1, gives us the following equations; 


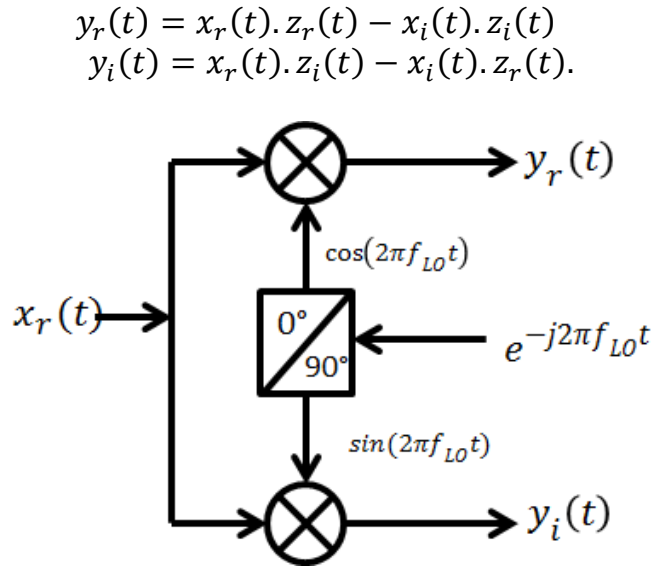

Fig. 1 Mixing real signal with complex exponential

The frequency transfer function of the polyphase filter is not symmetrical to the zero frequency. As such this characteristic of PPF is used in transceiver circuits to implement frequency mixing to reduce the non-idealities in the image signal. It is also used to produce balance quadrature signal at the output.

The $90^{\circ}$ hybrid is implemented using a lumped element passive RC polyphase filter. Polyphase filters are also known as complex filters. Their magnitude response is a function of both the input frequency and phase. The input of the complex filter represents the real and imaginary part of the signal. In RF receiver/transceiver the signal is created by separating the I (In-phase) and Q (Quadrature) components through the process of down conversion. Image rejection can be implemented using a polyphase filter [25].

Quadrature generation is widely used in many RF transceiver designs. It is important to consider the accuracy to generate $90^{\circ}$ phase shift signal since it is the key attribute to determine the image rejection ratio (IRR) which is equally important to define the transceiver sensitivity [9]. There are different techniques to generate a quadrature shift signal, e.g., frequency division, Haven's technique, RC-CR network and RC-PPF networks. In this design, the RCPolyphase network is adopted to perform quadrature phase shift.

\section{POLYPHASE FILTER}

In theory, polyphase filter can cancel the image signal. An image signal is any frequency other than the selected radio frequency carrier that, if allowed to enter the transceiver it will mix with the oscillator and produce a crossed product frequency that is equal to the IF signal. The simple series connection of RC sub-networks that is composed of a ring of resistors and capacitors is arranged alternately to help eliminate the image signal. A simple PPF has 4 inputs and 4 outputs. The phase difference between each port is $90^{\circ}$. The main purpose of this architecture is to generate four (4) quadrature signals that are required in image reject receiver or SSB modulators [20]. These receivers have the ability to pre-select and reject the image frequencies. The maximum image rejection depends on the performance of the PPF circuit, thus it is crucial to test its functionality and perform optimization prior to the system level design [18]. The accuracy of RC network will determine the filtering performance. One drawback of PPF when implemented on chip is its large chip size [21].

A single-Stage RC Polyphase filter is depicted in Fig. 2 contributing to one pole frequency called notch, where the image is attenuated around the area. It can be express as:

$$
f_{\text {pole }}=\frac{1}{2 \pi R C} .
$$

The transfer function $H(\omega)$ can be expressed as:

$$
H(\omega)=H_{1}(\omega)+j H_{2}(\omega)
$$

The positive sequence voltage transfer function can be written as:

$$
H(\omega)=A \frac{1-\omega R C}{1+j \omega R C}
$$

and the negative sequence voltage transfer function is written as:

$$
H(-\omega)=A \frac{1+\omega R C}{1+j \omega R C} .
$$

The characteristic gain of RC PPF depends on the combination of $\mathrm{RC}$ values and the magnitude of the zeros. Thus for PPF design, the value of $\mathrm{R}$ and $\mathrm{C}$, the order of the location of the zero and the magnitude of each zero must be taken into consideration [22].

The input and output I/Q equation is defined as

$$
\begin{aligned}
I_{\text {in }} & =I_{\text {in }+}-I_{\text {in }} \\
Q_{\text {in }} & =Q_{\text {in }+}-Q_{\text {in }} \\
I_{\text {out }} & =I_{\text {out }+}-I_{\text {out }} \\
Q_{\text {out }} & =Q_{\text {out }+}-Q_{\text {out }-}
\end{aligned}
$$

The output of the polyphase filter is a differential quadrature, $\mathrm{I}_{\text {out }}, \mathrm{I}_{\text {out }}, \mathrm{Q}_{\text {out }}$ and $\mathrm{Q}_{\text {out- }}$ respectively. PPF output is usually followed by a differential amplifier to improve the dynamic range.

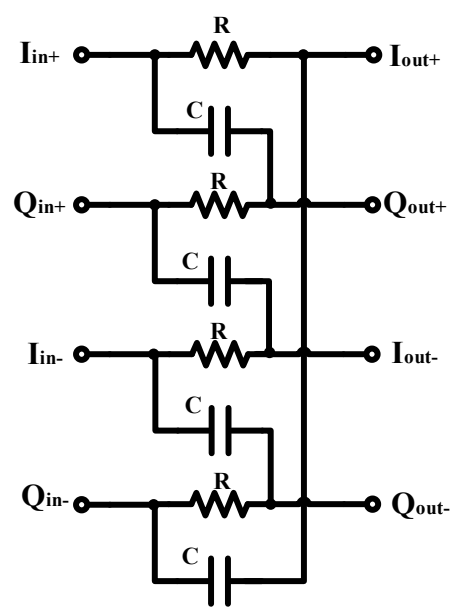

Fig. 2 Single-stage RC polyphase filter network 


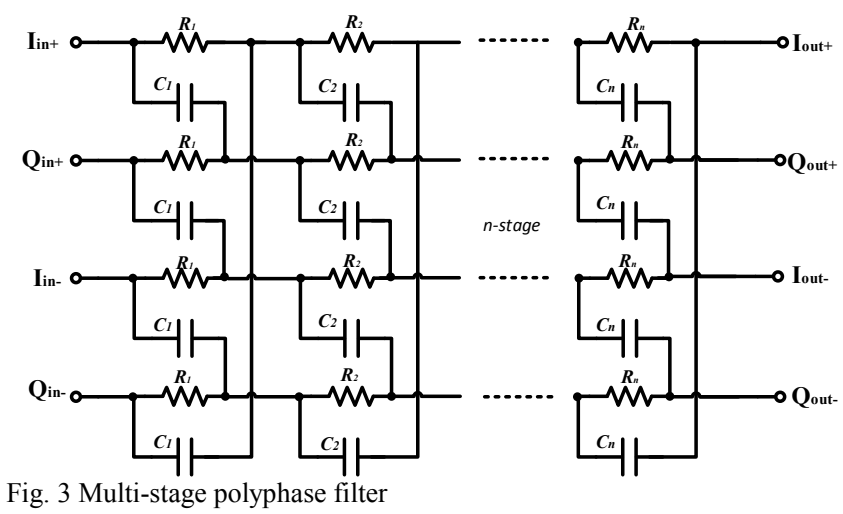

Fig. 3 shows the multiple stage polyphase filters, the cascading n-stage $\mathrm{RC}$ network can produce $0^{\circ}, 90^{\circ}, 180^{\circ}$ and $270^{\circ}$ respectively. By increasing the number of $\mathrm{RC}$ stages, we can improve the image rejection ratio (IRR). The required number of stages determines the bandwidth requirement of the channel. The PPF can provide good constant gain matching and quadrature precision. However, one drawback of the multi-stage design is the large layout area or chip footprint for wideband application [25].

A single PPF contributes one-pole frequency at which the image signal is attenuated; however it provides a narrowband rejection. A wider bandwidth can be accomplished by cascading a multi-stage PPF. The notches that the multi-stage PPF have are placed at the equal frequency ratio, given the following equation:

$$
\alpha=\frac{f_{p 2}}{f_{p 1}}=\frac{f_{p 3}}{f_{p 2}}=\frac{f_{p n}}{f_{p 3}} .
$$

Wherein the pole frequency ratios $\alpha$ are equal. For a better image rejection, a smaller $\alpha$ ratio is desired.

\section{A. PPF Excitation}

To make used of the four-phase I/Q signal, the input terminals must be connected in a proper way. There are two input feeding topologies or PPF excitations. Type I generates constant amplitude, the input asymmetric polyphase sequence consists a pair of phasors which have the same magnitude but phase difference of $180^{\circ}$. Type II has constant phase. The input asymmetric polyphase sequence is composed of two identical pairs of phasors where each pair has the same magnitude but they are separated by $180^{\circ}$. Type II excitation has a loss of about $3 \mathrm{~dB}$ less than type I [18][20][24].

The differential voltage can be express as

$$
\begin{aligned}
V_{I} & =V_{i_{\text {out }^{+}}}-V_{i_{\text {out }}} \\
V_{Q} & =V_{Q_{\text {out }^{+}}}-V_{Q_{\text {out }}}
\end{aligned}
$$

In type I the magnitude (A) and the phase $(\phi)$ is defined as;

$$
\begin{aligned}
& A=\frac{1}{|\omega R C|} \\
& \emptyset=-\frac{\pi}{2}
\end{aligned}
$$

In type II the magnitude (A) and the phase $(\phi)$ is defined as;

$$
\begin{aligned}
& A=1 \\
& \varnothing=\arctan \left(\frac{-2 \omega R C}{1-(\omega R C)^{2}}\right)
\end{aligned}
$$

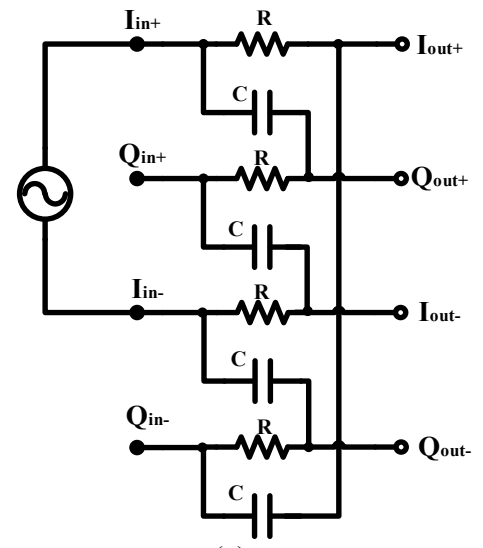

(a) Type I

(b)

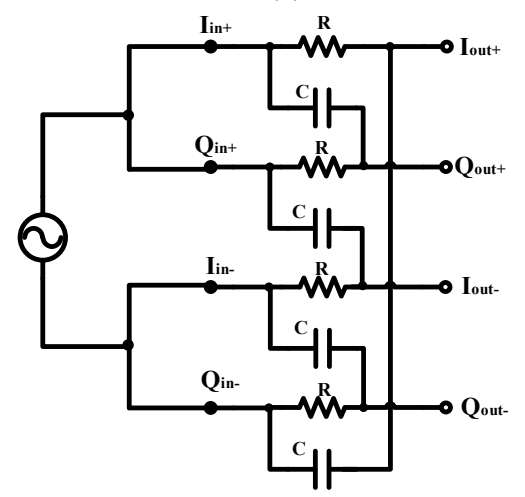

(b) Type II

Fig. 4 Polyphase filter input feeding excitation

\section{B. PPF Termination}

There are two ways to terminate a polyphase filter as discussed in [23]. The first method in Fig.5(a) shorted pairwise output, where $\mathrm{I}_{\text {out }}$ is shorted to $\mathrm{Q}_{\text {out }}$ and $\mathrm{Q}_{\text {out- is }}$ is shorted to $\mathrm{I}_{\text {out-, }}$ respectively. In the second method Fig.5(b) one output pair termination in dummies, where the differential output signal from $\mathrm{IQ}_{\text {out }}$ and $\mathrm{IQ}_{\text {out- }}$ is terminated using a dummy capacitance.

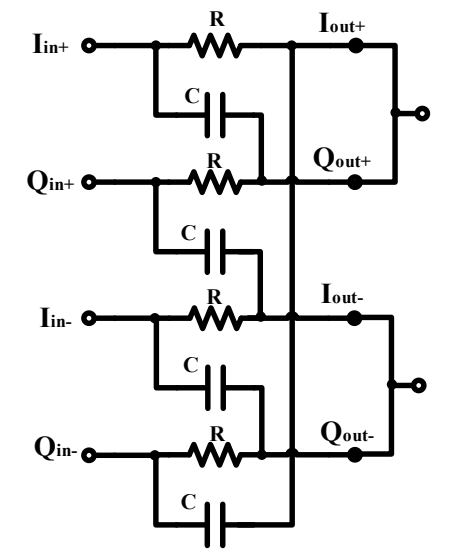

Fig.5(a) Shorted pairwise output termination 


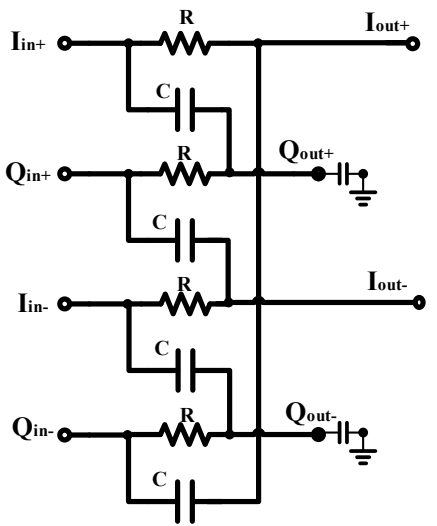

Fig. 5(b) One output pair termination

\section{SIMULATIONS}

As shown in Fig. 6, the output of each of the I/Q port of the PPF shifter is in quadrature with respect to other branch. The amplitude of the output sinusoidal signals are equal. Fig. 7 shows the simulation of the one stage polyphase filter generating $90^{\circ}$ and $-90^{\circ}$ phase difference from each I/Q branch. Fig. 8 shows the 3 stage PPF I/Q out phase Iout + , Qout + , Iout-, Qout + , and generate $0^{\circ}, 90^{\circ}$, $180^{\circ}$ and $270^{\circ}$ phase difference from each I/Q output branch respectively.

A multi-stage PPF is depicted in Fig.3. By cascading several stages with different notch frequency, a wide bandwidth can be achieved. Simulation of the phase of the I/Q signal is shown in Fig. 7, for 1, 2 and 3 stages respectively and Fig. 8 for 4,5 and 6 stages respectively. As can be seen, the higher number of the cascading stages, the higher the bandwidth generated by the PPF increases. The bandwidth and the percent increase of each stage are summarizing in Table 1. At 2 stage cascaded PPF, the bandwidth is $0.4 \mathrm{GHz}$, at 3 stages PPF the bandwidth is $1.517 \mathrm{GHz}$ which is $73.6 \%$ increase in bandwidth as compare to 2 stages PPF. Thus, the required number of the stages determines the bandwidth requirement of the channel. One trade-off of multi-stage PPF is the gain losses generated at previous stage.

A comparison with other polyphase filter designs is given in table 2. Note that the PPF listed are implemented in 4-stages, in these paper the proposed design PPF achieved $2.3 \mathrm{GHz}$ bandwidth.

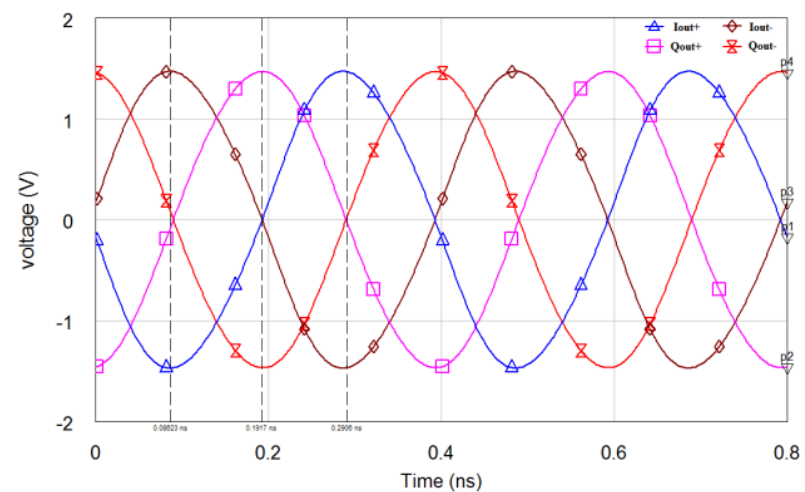

Fig. 6 Output voltage of 3-stage polyphase shifter with ideal differential source with respect to time

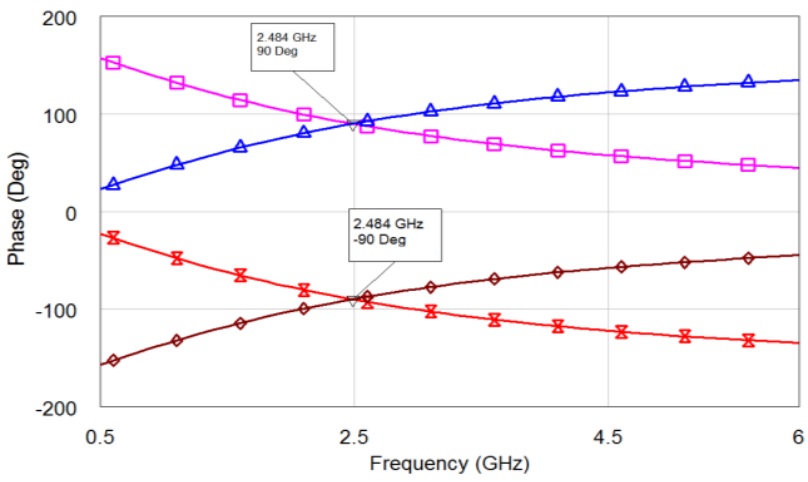

Fig.7 One stage $90^{\circ}$ phase difference between I/Q output branch

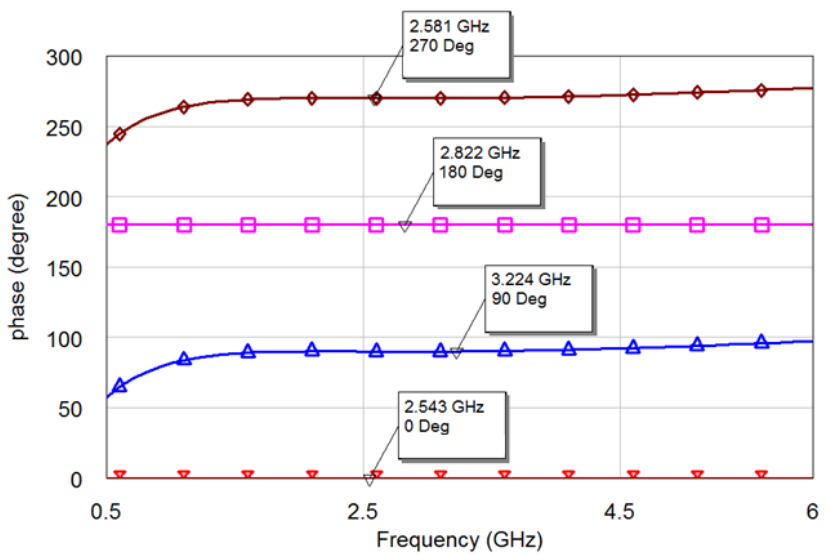

Fig.8 3-stage PPF I/Q output

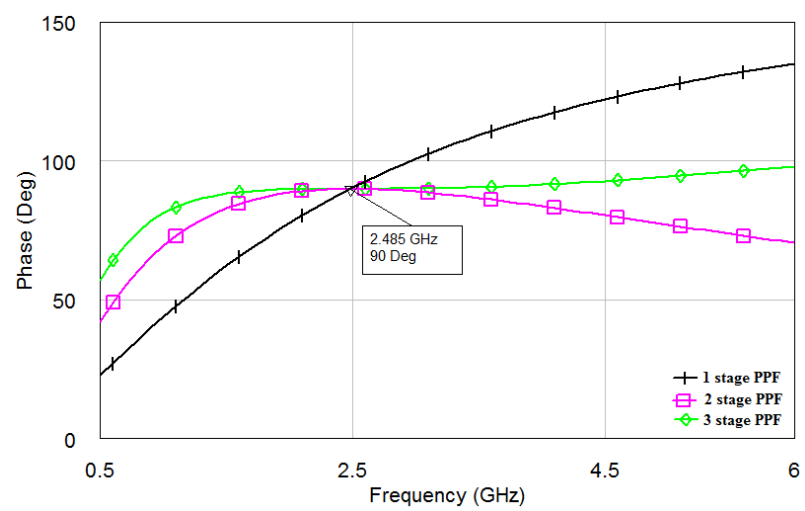

Fig. 9 Simulated phase of the I/Q signal of multi-stage PPF (1,2,3-stages respectively)

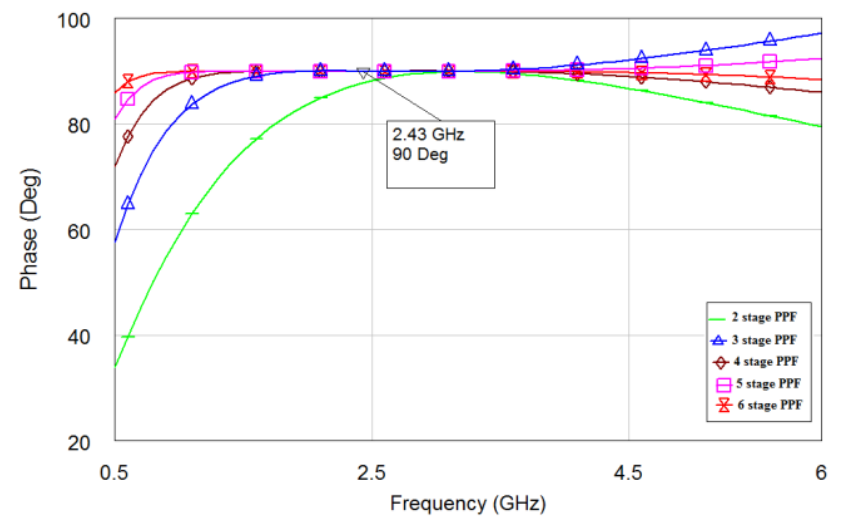

Fig. 10 Simulated phase of the I/Q signal of multi-stage PPF $(2,3,4,5$ and 6 - stages respectively) 
Table1. Summary of the multi-stage bandwidth

\begin{tabular}{|c|c|c|c|}
\hline Stage & Frequency range $\left(90^{\circ}\right)$ & Bandwidth & $\begin{array}{c}\% \\
\text { increase }\end{array}$ \\
\hline 2 & $2.8 \mathrm{GHz}-3.2 \mathrm{GHz}$ & $0.4 \mathrm{GHz}$ & \\
\hline 3 & $2.033 \mathrm{GHz}-3.55 \mathrm{GHz}$ & $1.517 \mathrm{GHz}$ & $73.6 \%$ \\
\hline 4 & $1.405 \mathrm{GHz}-3.709 \mathrm{GHz}$ & $2.304 \mathrm{GHz}$ & $34.15 \%$ \\
\hline 5 & $1.129 \mathrm{GHz}-3.96 \mathrm{GHz}$ & $2.831 \mathrm{GHz}$ & $18.6 \%$ \\
\hline 6 & $0.95 \mathrm{GHz}-4.1 \mathrm{GHz}$ & $3.15 \mathrm{GHz}$ & $10.13 \%$ \\
\hline
\end{tabular}

Table 2 Comparison with previous work

\begin{tabular}{|c|c|c|c|c|c|}
\hline Ref. & {$[5]$} & {$[21]$} & {$[26]$} & {$[27]$} & This work \\
\hline Process & CMOS & CMOS & CMOS & CMOS & BiCMOS \\
$(\mu \mathrm{m})$ & 0.13 & 0.35 & 0.13 & 0.25 & 0.13 \\
\hline Topology & Passive & Active & Tunable & Active & Passive \\
& RC & RC & & RC & RC \\
\hline Stages & 4 & 4 & 4 & 4 & 4 \\
\hline Bandwidth & $1.8 \mathrm{GHz}$ & $72 \mathrm{MHz}$ & $0.5 \mathrm{GHz}$ & $24 \mathrm{MHz}$ & $2.3 \mathrm{GHz}$ \\
\hline
\end{tabular}

\section{CONCLUSIONS}

This paper presents a multi-stage polyphase complex filter design to generate a wide bandwidth quadrature I/Q signal with constant $90^{\circ}$ phase shift for self-interference cancellation circuit for full duplex radio. By cascading nstages passive RC PPF an increase of $87 \%$ in bandwidth was attained by implementing 2 to extended 6 stages. A 4 stage PPF achieved $2.3 \mathrm{GHz}$ bandwidth. The broadband PPF was used to eliminate unwanted signal in RF front-end low-IF transceivers with interference cancellation mechanism. However, a multi-stage design has a trade-off with increase complexity, large chip footprints and gain loss.

\section{REFERENCES}

[1] H. Liu, X. Zhu, C. C. Boon and X. He, "Cell-based variable-gain amplifiers with accurate dB-linear characteristic in $0.18 \mu \mathrm{m}$ CMOS technology," IEEE J. Solid-State Circuits, vol. 50, pp. 586-596, 2015.

[2] H. Liu, X. Zhu, C. C. Boon and X. He, “A $71 \mathrm{~dB} 150 \mu \mathrm{W}$ variablegain amplifier in $0.18 \mu \mathrm{m}$ CMOS Technology," IEEE Microw. Wireless Compon. Lett., vol. 25, no. 5, pp. 334-336 2015.

[3] H. Liu, C. C Boon, X. He and X. Zhu, Y. Xiang, L. Kong and M. C. Heimlich, "A wideband analog-controlled variable-gain amplifier with dB-linear characteristic for high-frequency applications," IEEE Trans. Microw. Theory Tech., Vol. 64, No. 2, pp. 533-540, Feb. 2016

[4] X. He, X. Zhu, L. Duan, Y. Sun, C. Ma "A 14-mW PLL-less receiver in $0.18-\mu \mathrm{m}$ CMOS for Chinese electronic toll collection standard," IEEE Trans. Circuits Syst. II, Exp. Papers, vol. 61, no. 10 pp. 763-767, 2014. 10.1109/TCSII.2014.2345303

[5] F. Hadded, L.Zaid, W. Rahajandraibe, O.Frioui “ Polyphase Filter Design Methodology for Wireless Communication System” Mobile and Wireless Communication Network Layer and Circuit Level Design, Salma Ait fares and Fumiyuki Adachi (Ed.) ISBN: 978-953307-042-1, 2010, pp .220-246.

[6] Z.Zhang "Analysis, Design and Optimization of RF CMOS Poplyphase Filters" (Doctoral Dissertation), University of DuisburgEssen, Germany, 2005.

[7] A. Abidi " RF CMOS comes of Age", IEEE Journal of Solid-State Circuits, Vol. 39, No. 4, APRIL 2004, pp.549-561

[8] T.H. Lee "Recent Development in CMOS RF Integrated Circuits" Proceedings of the IEEE International Microwave Symposium (MTT-S'98), Baltimore, ML, 2008, pp.137-141.

[9] J.Crols, M.Steyaert "CMOS Wireless Transceiver Design”, Kluwer Academic Publisher, second edition, 2000.

[10] J.Choi, M.Jain, K.Srinivasan, P.Lewis, S.Katti “Achieving Single Channel Full Duplex Wireless Communication”, MobiCom'10 Proceedings of the Sixteenth Annual International Conference on Mobile Computing and networking, pp 1-12, September, 2010
[11] D.Bharadia, E.McMilin, S.Katti, "Full Duplex Radio" Special Interest Group On Data Communications, August 2013

[12] Y. Sun, X. Zhu, and J. Moritz, "Explicit design formulas for currentmode leap-frog OTA-C filters and $300 \mathrm{MHz}$ CMOS seventh-order linear phase filter," International Journal of Circuit Theory and Applications, ISSN: 0098-9886, Vol. 38, Issue: 4, p. 367-382, 2008.

[13] X. Zhu, and Y. Sun, "New low distortion low voltage operational transconductance amplifier," IET Electronics Letters, Vol. 44, No. 25, 2008.

[14] X. Zhu, Y. Sun, and J. Moritz, “A 0.18mm CMOS 300MHz currentmode LF seventh-order linear phase filter for hard disk read channels," IEEE International Symposium on Circuits and Systems (ISCAS), 2007.

[15] X. Zhu, Y. Sun and J. Moritz, “A CMOS 80mW 400MHz seventhorder MLF FLF linear phase filter with gain boost," International Conference on Electronics, Circuits and Systems (ICECS), 2008.

[16] X. Zhu, Y. Sun, and J. Moritz, "A CMOS fifth-order $400 \mathrm{MHz}$ current-mode LF linear phase filter for hard disk read channels," European Conference on Circuit Theory and Design (ECCTD), 2007.

[17] X. Zhu, Y. Sun and J. Moritz, "A CMOS 650MHz seventh-order current-mode $0.05^{\circ}$ equiripple linear phase filter," IEEE International Midwest Symposium on Circuits and Systems (MWSCAS), 2007.

[18] F.Haddad, W.Rahajandraibe, A.Slimane "Design of an Optimal Layout RF Passive Polyphase Filter for Large Image rejection" IEEE International Symposium on Circuits and Systems (ISCAS) May, 2015, pp. 269-272.

[19] Galal S.H, et al " RF sequence asymmetric polyphase network for RF integrated transceivers", IEEE Trans. Circuits and Systems II, 2000, pp $1127-1134$.

[20] A.Leggieri, D.Passi, D.gagliesi, F.D.Paolo “ Analysis and Design of a GaAs Monolithic Tunable Polyphase Filter in S/C Bands" Journal of Microwave Optoelectric and Electromagnetic Applications, Vol. 14 , no. 1, June 2015, pp. 14-27.

[21] S.C.Hsia, K.T.Lin "High Performance Active Polyphase Filter Design for Digital TV tunner" Microelectronics Journal, vol. 40, Issue 6, 2009, pp.966-972.

[22] N.Takai, Y.Sugawara, K.Suzuki, T.Negishi, H.Seki, M.Kato "Automatic Design of RF Polyphase Filter with Small Element Value Spread Using Genetic Algorithm”, International Symposium on Intelligent Signal processing and Communication System (ISPACS), Nov. 2015, pp. 303-306.

[23] F. Behbahani, Y.Kishigami, J.Leete, A.Abidi " CMOS Mixer and Polyphase Filter for Large Image rejection" IEEE Journal of SolidState Circuits, Vol. 36, No. 6, June 2001, pp. 873-887.

[24] V. Vidojkovic, J.V.D.Tang, A.Leeuwenbyrgh, A.V.Roermund "Adaptive Multi-standard RF Front-Ends" Analog and Signal Processing Series, Springer, ISBN 978-1-4020-6533-0 Springer Publication, 2008, pp. 53-77.

[25] S.T.Noall, "A WideBand Precision Quadrature Phase Shifter" (Master Thesis), Brigham Young University, Provo, USA, 2011.

[26] F.Haddad, W.Rahajandraibe, L.Zaid, O.Frioui, R.Bouchakour, "Design of Radio Frequency Passive Polyphase filter for $2.4 \mathrm{GHz}$ Wireless Communication Applications", IEEE Wireless and Microwave Technology Conference, 2009, pp.1-4.

[27] C.Y.Chou, C.Y.Wu, "The Design of Wideband and low power CMOS Active polyphase filter and its application in RF quadrature receivers", IEEE Transaction on Circuits and Systems-I, Regular Paper, Vol.52, No.5, May 2005, pp. 825-833.

[28] S. Chakraborty, et al, "A broadside-coupled meander-line resonator in $0.13-\mu \mathrm{m} \mathrm{SiGe} \mathrm{technology} \mathrm{for} \mathrm{millimeter-wave} \mathrm{application,"} \mathrm{IEEE}$ Electron Devices Lett., Vol. 37, No. 3, pp. 329-331, Mar. 2016. $\underline{10.1109 / \text { LED.2016.2520960 }}$ 